\title{
On the Total Variation of High-Order Semi-Discrete Central Schemes for Conservation Laws
}

\author{
Steve Bryson ${ }^{1}$ and Doron Levy ${ }^{2}$
}

Received October 14, 2004; accepted (in revised form) March 16, 2005; Published online March 13, 2006

\begin{abstract}
We discuss a new fifth-order, semi-discrete, central-upwind scheme for solving one-dimensional systems of conservation laws. This scheme combines a fifthorder WENO reconstruction, a semi-discrete central-upwind numerical flux, and a strong stability preserving Runge-Kutta method. We test our method with various examples, and give particular attention to the evolution of the total variation of the approximations.
\end{abstract}

KEY WORDS: High-order; central schemes; conservation laws; total variation. AMS(MOS) SUBJECT CLASSIFICATION. 65M06.

\section{INTRODUCTION}

In this paper we present a fifth-order, essentially non-oscillatory central-upwind scheme that is designed to solve systems of conservation laws of the form

$$
q_{t}+f(q)_{x}=0
$$

Here $q \in \mathbb{R}^{p}$ is a $p$-dimensional solution vector and $f$ is a $p$-dimensional flux function. The solution of (1.1) may become singular in finite time, which in turn requires a careful study when dealing with numerical approximations.

One approach to approximating solutions of (1.1) is to use high-order, non-oscillatory central methods, which were introduced in [15]. Central methods avoid approximating the solution of (1.1) at singularities of the

\footnotetext{
${ }^{1}$ NASA Advanced Supercomputing Division, NASA Ames Research Center, Moffett Field, CA 94035-1000, USA. E-mail: bryson@nas.nasa.gov

${ }^{2}$ Department of Mathematics, Stanford University, Stanford, CA 94305-2125, USA. E-mail: dlevy@math.stanford.edu
} 
solution, and so do not require solving Riemann problems. The resulting simplicity makes central schemes well-suited for systems and multiple dimensions. Central-upwind schemes, introduced in [7] and refined in [5], are semi-discrete variants of central methods which have improved efficiency and less dissipation than fully-discrete central methods. Our work uses the numerical flux of [5], which we refer to as the KNP flux.

In this work we combine the KNP flux with the fifth-order weighted essentially non-oscillatory (WENO) reconstruction of [3], and the fivestage fourth-order strong stability preserving (SSP) Runge-Kutta method of [19], which is based on [1]. This is the first time these particular ingredients are combined into one scheme.

Fourth-order fully-discrete central schemes based on WENO reconstructions were presented in $[10,13]$. The total variation behavior of these methods was examined in [12], where numerical experiments suggest that though the WENO-based methods are not total variation diminishing (TVD), they are total variation bounded. Fifth- and ninth-order fully-discrete central schemes are discussed in [16]. Third-order extensions of the KNP scheme can be found in $[4,6]$.

In this paper, we investigate the evolution in time of the total variation (TV) of our scheme. The TV is defined for a discrete solution $u_{j}$ as $\mathrm{TV}(u):=\sum_{j}\left|u_{j+1}-u_{j}\right|$. In the case of systems TV is defined as the sum of the TV over the components. A scheme is called TV bounded (TVB) in $0 \leqslant t \leqslant T$ if $\mathrm{TV}(u) \leqslant K$ for fixed $K>0$ which depends only on initial conditions, and $\forall n$ and $\forall \Delta t \leqslant \Delta t_{0}$ such that $n \Delta t \leqslant T$ and $\Delta t_{0}$ is provided by the stability requirement. In the scalar case, if a scheme is TVB then there exists a convergent subsequence in $L_{\text {loc }}^{1}$ to a weak solution of (1.1), which turns into strong convergence if an additional entropy condition is satisfied (see [9]). Our numerical experiments suggest that our method is TVB, providing evidence of the convergence of the method.

The structure of this paper is as follows: in Sec. 2 we present our fifth-order central-upwind scheme, summarizing the derivation of the KNP flux in Sec. 2.1. The WENO reconstruction is summarized in Sec. 2.2. Section 3 presents the results of a number of numerical tests of our method. We test both the accuracy and the evolution of the total variation of the resulting approximations.

\section{THE NUMERICAL SCHEME}

We briefly summarize the components we use to construct our fifthorder central-upwind scheme: the numerical flux from [5], and the reconstruction from [3]. 


\subsection{The KNP Flux}

Throughout this section, we assume a one-dimensional grid $\left\{x_{j}\right\}$ with constant spacing $\Delta x$. We define $x_{j \pm \frac{1}{2}}:=x_{j} \pm \frac{1}{2} \Delta x$ and the cell $I_{j}=$ $\left[x_{j-\frac{1}{2}}, x_{j+\frac{1}{2}}\right]$. For any function $f(x)$ we use the notation $f_{j}:=f\left(x_{j}\right)$. The cell average of $q$ in the cell $I_{j}$ is given by $\bar{q}_{j}:=\frac{1}{\Delta x} \int_{x_{j-\frac{1}{2}}}^{x_{j+\frac{1}{2}}} q(x) d x$.

We assume that the cell-averages $\bar{q}_{j}^{n}$ are known at time $t^{n}$. The first step in the derivation of the approximate solution is to generate a piecewise-polynomial reconstruction from these cell-averages. Such a global reconstruction is defined as $\tilde{q}(x)=\sum_{j} \tilde{q}_{j}(x) \chi_{I_{j}}(x)$, where $\chi_{I_{j}}(x)$ is the characteristic function of $I_{j}$, and $\tilde{q}_{j}(x)$ are polynomials of a suitable degree.

In each cell $I_{j}$ the reconstruction $\tilde{q}_{j}(x)$ should be conservative, i.e. $\frac{1}{\Delta x} \int_{x_{j-\frac{1}{2}}}^{x_{j+\frac{1}{2}}} \tilde{q}_{j}(x) d x=\bar{q}_{j}$, formally $s$ th-order accurate, (so $\tilde{q}_{j}(x)=q(x)+$ $O\left(\Delta x^{s}\right)^{2}$ for sufficiently smooth $q$ and $\left.x \in I_{j}\right)$, and non-oscillatory. Given such a reconstruction, we denote the point-values of $\tilde{q}$ at the interfaces of the cell $I_{j}$ by $q_{j+\frac{1}{2}}^{+}:=\tilde{q}_{j+1}\left(x_{j+\frac{1}{2}}\right)$ and $q_{j+\frac{1}{2}}^{-}:=\tilde{q}_{j}\left(x_{j+\frac{1}{2}}\right)$.

The left- and right-sided local speeds of propagation of information from the discontinuities at the cell interfaces, $a_{j+\frac{1}{2}}^{ \pm}$, are estimated by

$$
\begin{aligned}
& a_{j+\frac{1}{2}}^{+}=\max _{u \in C\left(q_{j+\frac{1}{2}}^{-}, q_{j+\frac{1}{2}}^{+}\right)}\left(\lambda_{N}\left(\frac{\partial f}{\partial q}(u)\right), 0\right), \\
& a_{j+\frac{1}{2}}^{-}=\left|\min _{u \in C\left(q_{j+\frac{1}{2}}^{-}, q_{j+\frac{1}{2}}^{+}\right)}\left(\lambda_{1}\left(\frac{\partial f}{\partial q}(u)\right), 0\right)\right| .
\end{aligned}
$$

Here, $\lambda_{1}<\cdots<\lambda_{N}$ denote the $N$ eigenvalues of the Jacobian of $f$ and $C\left(q_{j+\frac{1}{2}}^{-}, q_{j+\frac{1}{2}}^{+}\right)$is the curve in phase space connecting $q_{j+\frac{1}{2}}^{-}$and $q_{j+\frac{1}{2}}^{+}$. These local speeds of propagation are then used to determine intervals for averaging that contain the Riemann fans from the cell interfaces. An exact evolution of the reconstruction is followed by an intermediate piecewise polynomial reconstruction and finally projected back onto the original cells, providing the cell-averages at the next time-step $\bar{q}_{j}^{n+1}$. Further details can be found in [5]. A semi-discrete scheme is obtained in the limit 
as $\Delta t \rightarrow 0$, yielding the KNP central-upwind scheme

$$
\frac{d \bar{q}_{j}}{d t}=-\frac{H_{j+\frac{1}{2}}-H_{j-\frac{1}{2}}}{x_{j+\frac{1}{2}}-x_{j-\frac{1}{2}}}
$$

The numerical flux in (2.1) is given by

$$
H_{j+\frac{1}{2}}=\frac{a_{j+\frac{1}{2}}^{+} f\left(q_{j+\frac{1}{2}}^{-}\right)+a_{j+\frac{1}{2}}^{-} f\left(q_{j+\frac{1}{2}}^{+}\right)}{a_{j+\frac{1}{2}}^{+}+a_{j+\frac{1}{2}}^{-}}-\frac{a_{j+\frac{1}{2}}^{+} a_{j+\frac{1}{2}}^{-}}{a_{j+\frac{1}{2}}^{+}+a_{j+\frac{1}{2}}^{-}}\left[q_{j+\frac{1}{2}}^{+}-q_{j+\frac{1}{2}}^{-}\right] .
$$

The accuracy of this scheme is determined by the accuracy of the reconstructions and the ODE solver.

It is straightforward to generalize this scheme to higher dimensions, using dimension-by-dimension reconstructions. Care must be taken, however, to use higher-order quadratures in the derivation of the KNP flux in higher dimensions to maintain accuracy. See [6] for a third-order example.

\subsection{The Fifth-Order WENO Reconstruction}

Weighted, essentially non-oscillatory (WENO) reconstructions [3, 14] are based on the essentially non-oscillatory (ENO) reconstructions of [2, 17]. ENO schemes choose the stencil that provide the least oscillatory reconstruction. WENO schemes weight all stencils so that accuracy is gained in smooth regions while trying to avoid crossing discontinuities.

We use the fifth-order WENO reconstruction of the point-value $q_{j+\frac{1}{2}}$ given in [3]. We begin with the three quadratic reconstructions on threepoint stencils

$$
q_{j+\frac{1}{2}}^{k}=\sum_{r=0}^{2} a_{r}^{k} \bar{q}_{j+k+r-2}
$$

where $k$ ranges from 0 to 2 and the coefficients $a_{r}^{k}$, given in Table I, are defined so that (2.2) approximates $q\left(x_{j+\frac{1}{2}}\right)$ with third-order accuracy. The WENO reconstruction is then defined as the convex combination

$$
q_{j+\frac{1}{2}}=\sum_{k=0}^{2} w_{j}^{k} q_{j+\frac{1}{2}}^{k}, \quad w_{j}^{k}:=\frac{\alpha_{j}^{k}}{\sum_{k=0}^{2} \alpha_{j}^{k}}, \quad \alpha_{j}^{k}:=\frac{C^{k}}{\left(\epsilon+S_{j}^{k}\right)^{2}}
$$


Table I. Coefficients $a_{r}^{k}$ for the Quadratic Reconstructions

$(2.2)$

\begin{tabular}{cccc}
\hline $\mathrm{k}$ & $r=0$ & $r=1$ & $r=2$ \\
\hline 0 & $1 / 3$ & $-7 / 6$ & $11 / 6$ \\
1 & $-1 / 6$ & $5 / 6$ & $1 / 3$ \\
2 & $1 / 3$ & $5 / 6$ & $-1 / 6$ \\
\hline
\end{tabular}

The constants $C^{k}=\{1 / 10,6 / 10,3 / 10\}$ are defined so that $\sum_{k=0}^{2} C^{k} q_{j+\frac{1}{2}}^{k}$ approximates $q\left(x_{j+\frac{1}{2}}\right)$ with fifth-order accuracy. $S_{j}^{k}$ is a smoothness measure which is large when $q_{j+\frac{1}{2}}^{k}$ has large variation. $S_{j}^{k}$ approximates the $L_{\text {loc }}^{2}$-norm of the first two derivatives of $q$, and is given by

$$
\begin{aligned}
S_{j}^{0} & =\frac{13}{12}\left(\bar{q}_{j-2}-2 \bar{q}_{j-1}+\bar{q}_{j}\right)^{2}+\frac{1}{4}\left(\bar{q}_{j-2}-4 \bar{q}_{j-1}+3 \bar{q}_{j}\right)^{2}, \\
S_{j}^{1} & =\frac{13}{12}\left(\bar{q}_{j-1}-2 \bar{q}_{j}+\bar{q}_{j+1}\right)^{2}+\frac{1}{4}\left(\bar{q}_{j-1}-\bar{q}_{j+1}\right)^{2}, \\
S_{j}^{2} & =\frac{13}{12}\left(\bar{q}_{j}-2 \bar{q}_{j+1}+\bar{q}_{j+2}\right)^{2}+\frac{1}{4}\left(3 \bar{q}_{j}-4 \bar{q}_{j+1}+\bar{q}_{j+2}\right)^{2} .
\end{aligned}
$$

Following [3] we take $\epsilon=10^{-6}$. The reconstruction of $q_{j-\frac{1}{2}}$ on the stencil centered at $x_{j}$ is obtained by symmetry. For more details consult [3].

\section{NUMERICAL EXAMPLES}

We test our method on various examples, measuring both the accuracy of the approximation and the evolution of the TV over time. To integrate (2.1) forward in time, we use an optimal, strong stability preserving fourthorder accurate five-stage Runge-Kutta solver from [19]. We also ran our examples with the standard Runge-Kutta method and observed no significant change in the results. We use the CFL condition $\Delta t=0.45 \min _{j} \frac{\Delta x}{\left|\lambda_{j}\right|}$, where here $\lambda_{j}$ denote the eigenvalues of the Jacobian of $f$ evaluated at $x_{j}$.

When an exact solution is not available, we use a high-resolution reference solution computed using the second-order KNP method as presented in [5], which is known to be TVD. Unless otherwise stated, this reference solution uses $N=5000$ nodes.

We first test our method with the scalar advection problem $u_{t}+u_{x}=$ $0, u(x, t=0)=\sin ^{4}(\pi x)$ on the periodic domain $[-1,1]$ at $T=2$. The relative $L^{1}$ - and $L^{\infty}$ - norms of the errors are shown in Table II. We also test 
Table II. Relative $L^{1}$ and $L^{\infty}$ Errors for the Advection Equation and the Burgers Equation

\begin{tabular}{ccccc}
\hline & Relative $L^{1}$-error & $L^{1}$-order & Relative $L^{\infty}$-error & $L^{\infty}$-order \\
\hline$N$ & Linear advection of $\sin ^{4}(\pi x), T=2$ \\
100 & $8.68 \times 10^{-4}$ & - & $1.14 \times 10^{-3}$ & \\
200 & $2.55 \times 10^{-5}$ & 5.09 & $4.18 \times 10^{-5}$ & - \\
400 & $6.32 \times 10^{-7}$ & 5.34 & $9.77 \times 10^{-7}$ & 5.77 \\
800 & $1.46 \times 10^{-8}$ & 5.44 & $1.49 \times 10^{-8}$ & 6.03 \\
& Burgers equation before the shock, $T=0.5$ \\
100 & $1.03 \times 10^{-6}$ & - & $7.51 \times 10^{-6}$ & - \\
200 & $3.87 \times 10^{-8}$ & 4.74 & $2.89 \times 10^{-7}$ & 4.70 \\
400 & $1.15 \times 10^{-9}$ & 5.08 & $8.12 \times 10^{-9}$ & 5.15 \\
800 & $8.87 \times 10^{-11}$ & 3.69 & $3.31 \times 10^{-10}$ & 4.61 \\
& Burgers equation after the shock, $T=2.5$ & \\
100 & $3.34 \times 10^{-3}$ & - & $1.42 \times 10^{-1}$ & - \\
200 & $1.99 \times 10^{-3}$ & 0.75 & $2.12 \times 10^{-1}$ & -0.57 \\
400 & $8.72 \times 10^{-4}$ & 1.19 & $1.65 \times 10^{-1}$ & 0.36 \\
800 & $4.38 \times 10^{-4}$ & 0.99 & $1.63 \times 10^{-1}$ & 0.01 \\
\hline
\end{tabular}

accuracy with the Burgers equation $u_{t}+\left(\frac{u^{2}}{2}\right)_{x}=0, u(x, t=0)=3+\sin (x)$ on the periodic domain $[0,2 \pi]$ at $T=0.5$, before shock formation, and at $T=2.5$ after shock formation. The results are also shown in Table II. Figure 1 shows the result at $T=2.5$, as well as the change in the TV over time for various resolutions. For this example the exact TV equals to 4 before the singularity formation at $T=1$.

Our next example is Burgers equation on the same domain with initial data $u(x, t=0)=2-\sin (x)+\sin (2 x)$. This example develops two shocks which eventually merge. Figure 2 shows the solution at $T=1.2$ as well as the change in the TV over time for various resolutions.

Turning to systems, we consider the Euler equations

$$
\left(\begin{array}{c}
\rho \\
\rho u \\
E
\end{array}\right)_{t}+\left(\begin{array}{c}
\rho u \\
\rho u^{2}+p \\
(E+p) u
\end{array}\right)_{x}=0
$$

with equation of state $p=(\gamma-1)\left(E-\frac{1}{2} \rho u^{2}\right)$ and $\gamma=1.4$. We first apply our method to the Lax problem [8] on the domain $[0,1]$ with initial data

$$
(\rho, u, E)= \begin{cases}(0.445,0.311,8.928), & x<0.5 \\ (0.5,0.0,1.4275), & x>0.5\end{cases}
$$

The results at $T=0.16$ with $N=100$ and $N=400$ grid nodes is shown in Fig. 3. While the shock and the contact discontinuity are well-captured at 

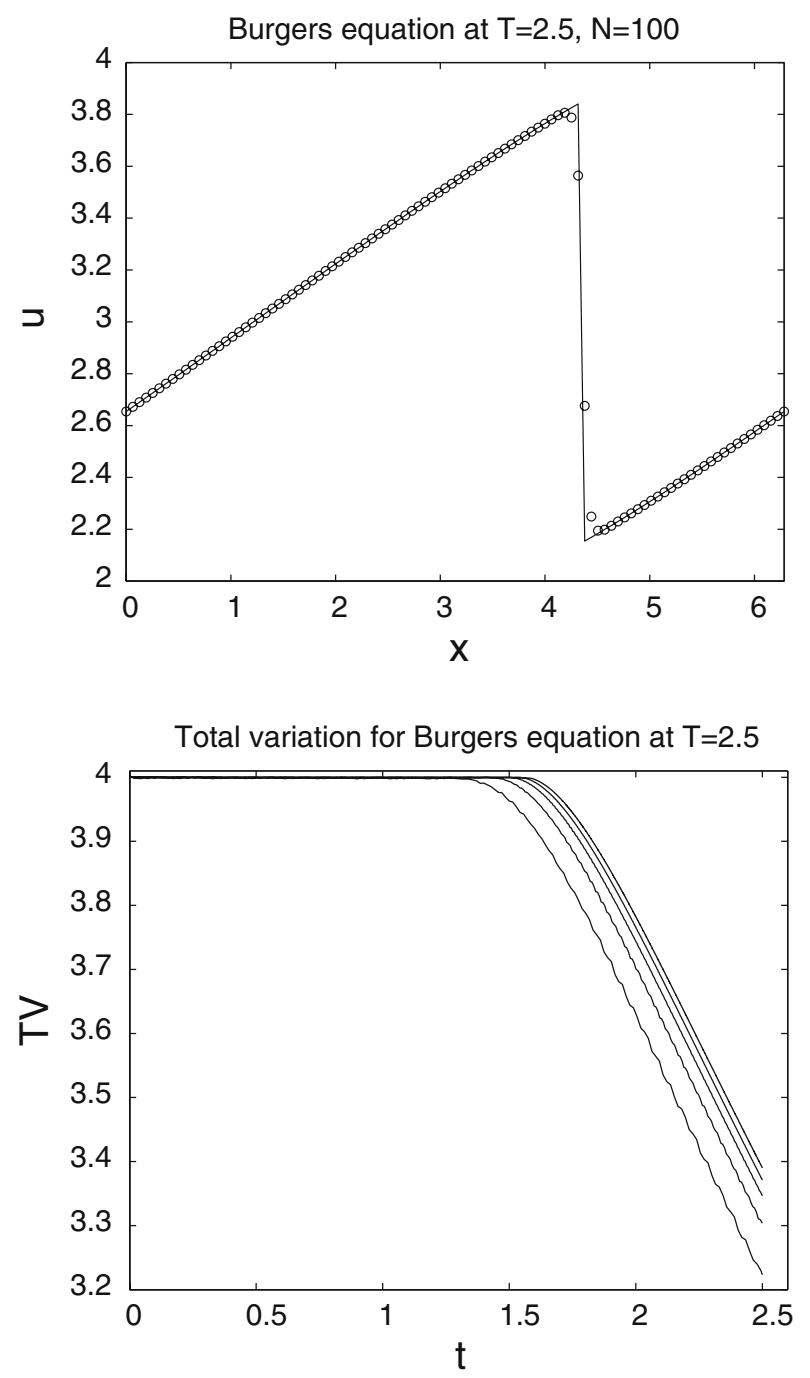

Fig. 1. Results for the Burgers equation using the central-upwind scheme (2.1) and (2.3). Top: the solution after shock formation at $T=2.5$, "-": exact solution, "o": approximation. Bottom: the change in the TV of the approximation for $N=100,200,400,800$ nodes (from left to right) compared with the TV of a reference solution (the upper curve). 

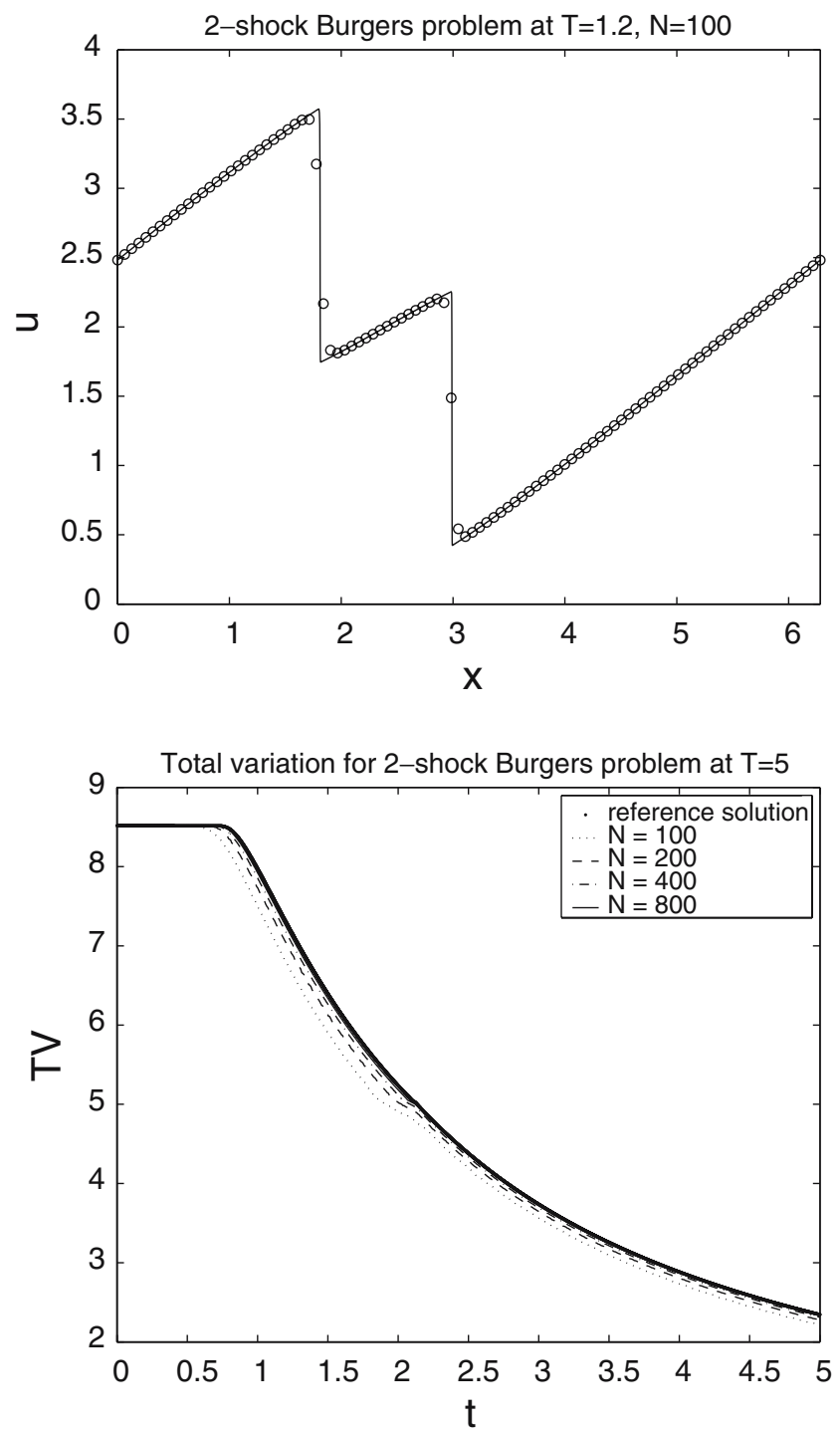

Fig. 2. Results for Burgers equation with initial data that develops a double shock using the central-upwind scheme (2.1) and (2.3). Top: the solution after shock formation at $T=1.2$, "-": exact solution, "o": approximation. Bottom: the change in the TV of the approximation for various resolutions compared with the TV of a reference solution. 
low resolution, there are significant oscillations between the contact discontinuity and the shock for $N=100$. Figure 3 also shows the TV behavior of the approximation, compared with a reference solution. We see that the TV of the approximate solutions are initially greater than that of the reference solution, but converges to the TV of the reference solution over time, with a faster rate of convergence for finer meshes. It is interesting, however, that the over-shoot of the TV at early times does not seem to depend on the mesh resolution. We observe similar behavior for the Sod problem [18].

We next apply our method to the reflected blast problem of Woodward and Colella [20], on the domain $[0,1]$ with reflecting boundary conditions and initial data

$$
(\rho, u, E)= \begin{cases}(1.0,0.0,2500.0), & 0 \leqslant x<0.1 \\ (1.0,0.0,0.01), & 0.1 \leqslant x<0.9, \\ (1.0,0.0,250.0), & 0.9 \leqslant x \leqslant 1 .\end{cases}
$$

The results at $T=0.038$ with $N=400$ grid nodes are shown in Fig. 4 , compared with a reference solution using 10,000 nodes. We see some numerical oscillations. Figure 4 also shows the TV behavior of the approximation, compared with the reference solution. We see that the TV of the approximate solutions converges to the TV of the reference solution for finer meshes, but do not seem to converge over time. This is not surprising since this example contains sharp peaks that will not be resolved for coarse meshes.

For our final example we apply our method to the problem of a mach three wave interacting with an acoustic shock on the domain $[0,1]$ (see [17]). The initial conditions for this problem are

$$
(\rho, u, p)= \begin{cases}(3.857143,2.629369,10.3333), & x \leqslant 0.1, \\ (1+0.2 \sin (50 x), 0,1), & x>0.1 .\end{cases}
$$

The results are shown in Fig. 5, compared with a reference solution using 20,000 nodes. We see the high resolution of our method and indications that the TV of our approximations converges to that of the reference solution.

In conclusion, we would like to note that the TV approaches the TV of the reference solution in different ways for different examples. While in some cases it is monotone (such as the acoustic-shock problem) in other cases it is not (such as the Woodward-Colella problem). Our results are characteristic of the complex structure that one may expect to find with the TV of solutions of systems (with the lack of any supporting theory). Trying to convert these observations into a statement on the convergence of the scheme remains an important topic for future study. 

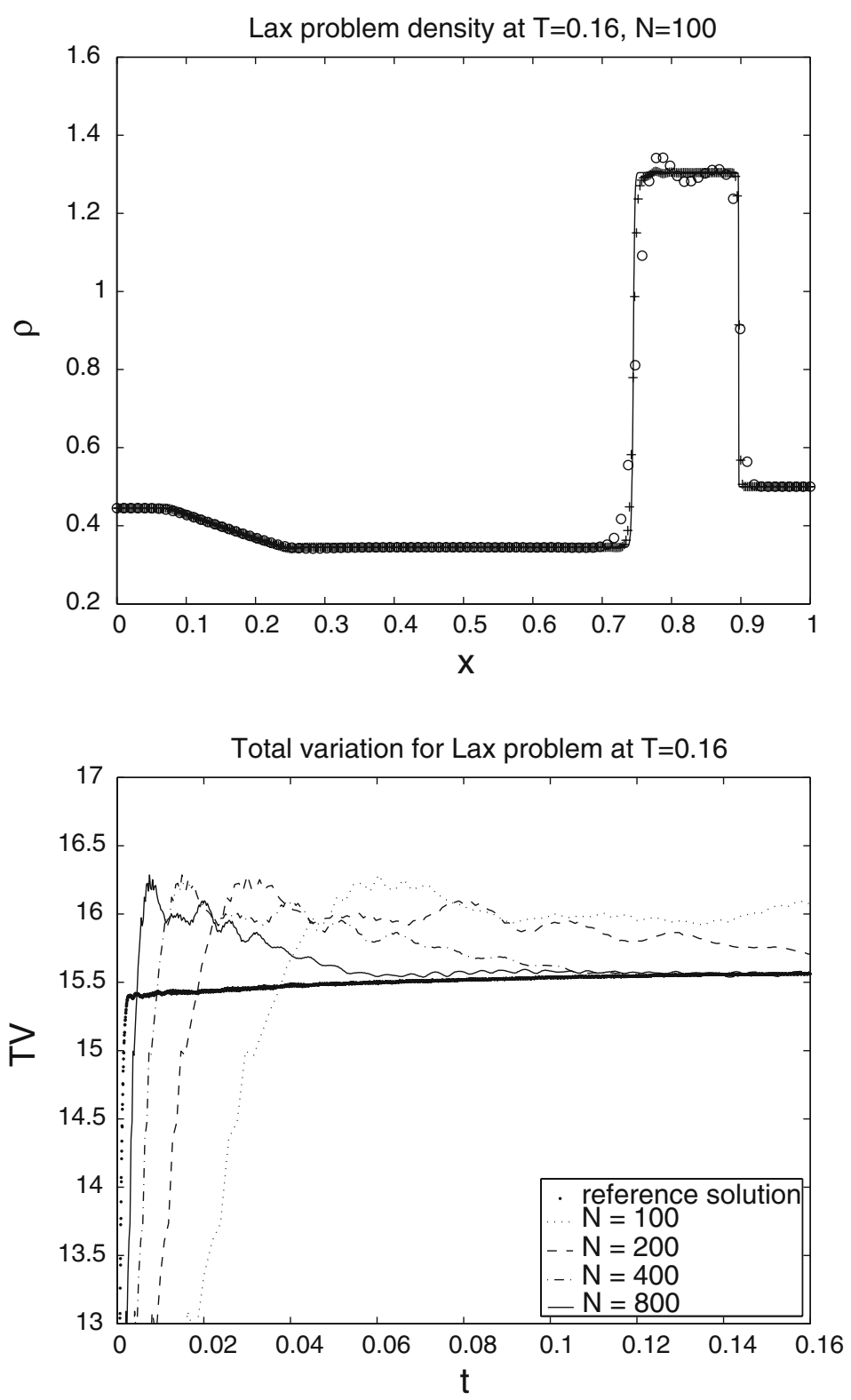

Fig. 3. Results for the Lax problem (3.2) using the central-upwind scheme (2.1) and (2.3). Top: Density. "-": reference solution, "o": approximation with $N=100$ nodes, "+": approximation with $N=400$ nodes. Bottom: the change in the TV of the approximation for various resolutions compared with the TV of a reference solution. 

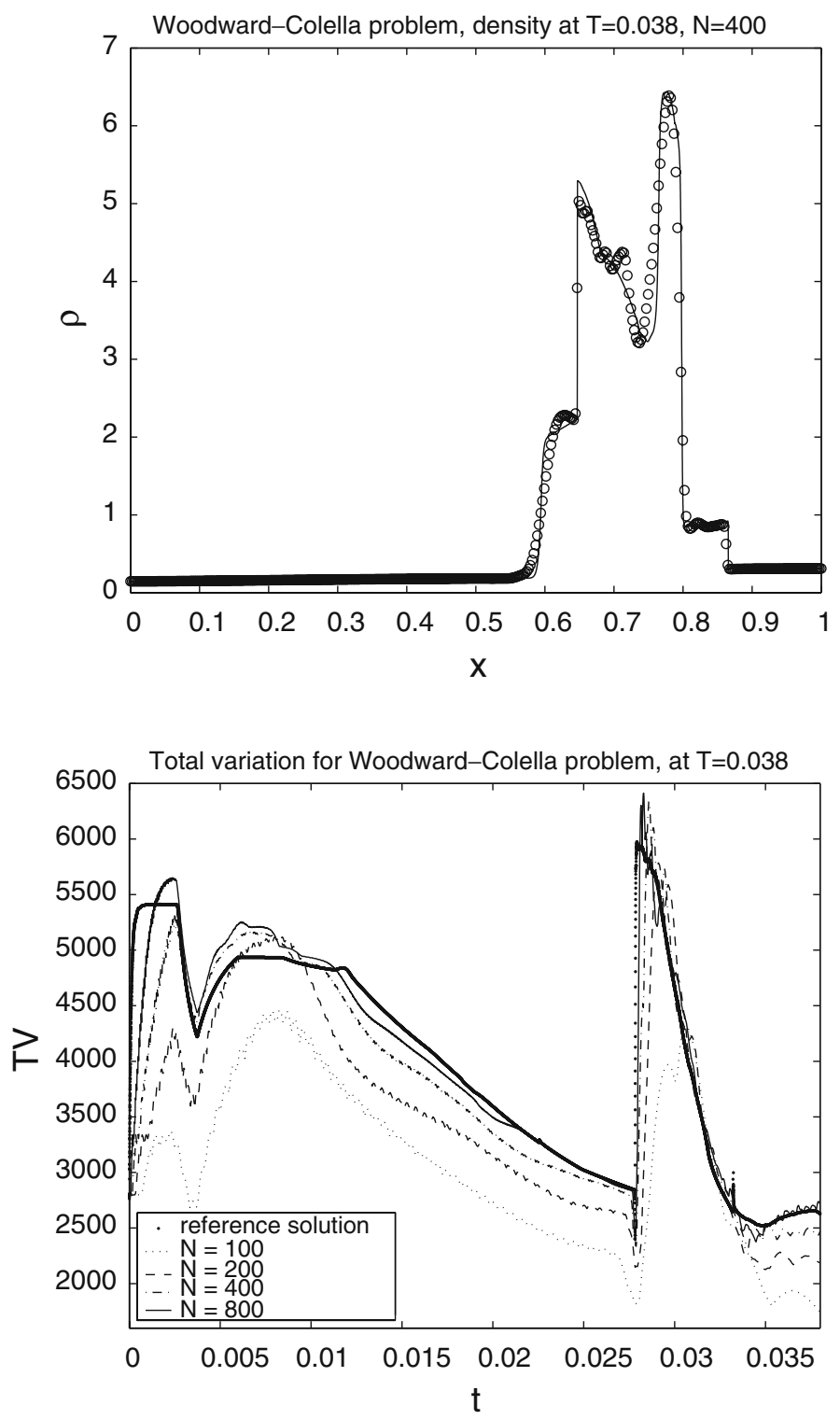

Fig. 4. Results for the Woodward-Colella problem (3.3) using the central-upwind scheme (2.1) and (2.3). Top: Density. "-": reference solution, "o": approximation with $N=400$ nodes. Bottom: the change in the TV of the approximation for various resolutions compared with the TV of a reference solution. 

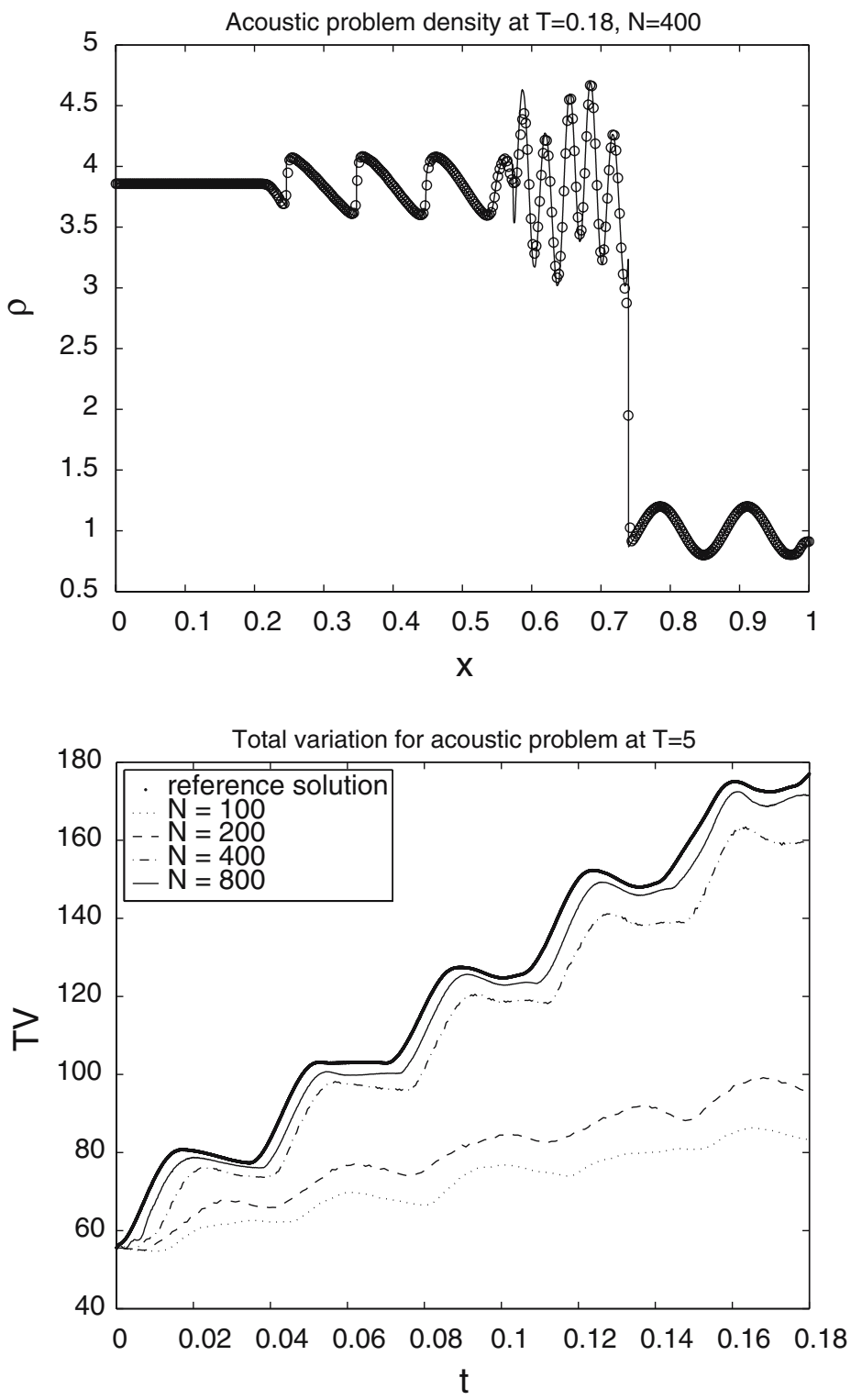

Fig. 5. Results for the acoustic-shock problem (3.4), showing the approximation of the density field at $T=0.18$ using $N=400$ nodes. Top: Approximate solution. "-": reference solution, "o": approximation. Bottom: the change in the TV of the approximation for various resolutions compared with the TV of a reference solution. 


\section{ACKNOWLEDGEMENTS}

The work of D. Levy was supported in part by the National Science Foundation under Career Grant No. DMS-0133511.

\section{REFERENCES}

1. Gottlieb, S., Shu, C.-W., and Tadmor, E. (2001). Strong stability-preserving high order time discretization methods. SIAM Rev. 43, 89-112.

2. Harten, A., Engquist, B., Osher, S., and Chakravarthy, S. (1987). Uniformly high order accurate essentially non-oscillatory schemes III. J. Comp. Phys. 71, 231-303.

3. Jiang, G.-S., and Shu, C.-W. (1996). Efficient implementation of weighted ENO schemes. J. Comp. Phys. 126, 202-228.

4. Kurganov, K., and Levy, D. (2000). A Third-order semi-discrete central scheme for conservation laws and convection-diffusion equations. SIAM J. Sci. Comp. 22, 1461-1488.

5. Kurganov, K., Noelle, S., and Petrova, G. (2001). Semi-discrete central-upwind schemes for hyperbolic conservation laws and Hamilton-Jacobi equations. SIAM J. Sci. Comp. 23, 707-740.

6. Kurganov, K., and Petrova, G. (2001). A Third-order semi-discrete genuinely multidimensional central scheme for hyperbolic conservation laws and related problems. Numer. Math. 88, 683-729.

7. Kurganov, A., and Tadmor, E. (2000). New high-resolution central schemes for nonlinear conservation laws and convection-diffusion equations. J. Comp. Phys. 160, 241-282.

8. Lax, P. D. (1954). Weak solutions of nonlinear hyperbolic equations and their numerical computation. Comm. Pure Appl. Math. 7, 159-193.

9. LeVeque, R. J. (1992). Numerical Methods for Conservation Laws, Lectures in Mathematics, Birkhuser, Basel.

10. Levy, D., Puppo, G., and Russo, G. (1999). Central WENO schemes for hyperbolic systems of conservation laws. Math. Model. Numer. Anal. 33, 547-571.

11. Levy, D., Puppo, G., and Russo, G. (2000). Compact central WENO schemes for multidimensional conservation laws. SIAM J. Sci. Comp. 22, 656-672.

12. Levy, D., Puppo, G., and Russo, G. (2000). On the behavior of the total variation in CWENO methods for conservation laws. Appl. Num. Math. 33, 415-421.

13. Levy, D., Puppo, G., and Russo, G. (2002). A fourth order central WENO scheme for multidimensional hyperbolic systems of conservation laws. SIAM J. Sci. Comp. 24, 480-506.

14. Liu, X.-D., Osher, S., and Chan, T. (1994). Weighted essentially non-oscillatory schemes. J. Comp. Phys. 115, 200-212.

15. Nessyahu, H., and Tadmor, E. (1990). Non-oscillatory central differencing for hyperbolic conservation laws. J. Comp. Phys. 87, 408-463.

16. Qiu, J., and Shu, C.-W. (2002). On the construction, comparison, and local characteristic decomposition for high order central WENO schemes. J. Comp. Phys. 183, 187-209.

17. Shu, C.-W., and Osher, S. (1988). Efficient implementation of essentially non-oscillatory shock-capturing schemes. J. Comp. Phys. 77, 439-471.

18. Sod, G. (1978). A survey of several finite difference methods for systems of nonlinear hyperbolic conservation laws. J. Comp. Phys. 27, 1-31.

19. Spiteri, R. J., and Ruuth, S. J. (2002). A new class of optimal high-order strong-stabilitypreserving time discretization methods. SIAM J. Numer. Anal. 40, 469-491.

20. Woodward, P., and Colella, P. (1984). The numerical simulation of two-dimensional fluid flow with strong shocks. J. Comp. Phys. 54, 115-173. 